\title{
Transformaciones más allá de la política pública de educación superior. El impacto de la formación profesional regionalizada de la Universidad de Antioquia*
}

DOI: https://doi.org/10.18046/recs.i34.4226

\author{
Transformations Beyond the Higher Education Public \\ Policy. The Impact of Universidad de Antioquia's Regionalized \\ Higher Education
}

\author{
Edinson Gabriel Brand-Monsalve ${ }^{* *}$ \\ Universidad de Antioquia (Medellín, Colombia) \\ Erika Yuliet Álvarez-Calle*** \\ Universidad de Antioquia (Medellín, Colombia) \\ Ingrid Yuranie Posso-Serna ${ }^{* * * *}$ \\ Universidad de Antioquia (Medellín, Colombia) \\ Julián Andrés Angarita-Suárez ${ }^{* * * *}$ \\ Universidad de Antioquia (Medellín, Colombia) \\ Katherine Londoño-Arbeláez ${ }^{* * * * *}$ \\ Universidad de Antioquia (Medellín, Colombia)
}

\footnotetext{
* Este artículo es resultado del proyecto de investigación "Medición del impacto de la educación superior regionalizada de la Universidad de Antioquia. Estudio de caso en los programas de Ingeniería, Sociología y Educación en la sede del Carmen", financiado por la Convocatoria Programática 2016 del Área de Ciencias Sociales, Humanidades y Artes de la Universidad de Antioquia (Colombia). Artículo de investigación recibido el 14.08.2O2O y aceptado el 24.06.2O21.

** Candidato a Doctor en Filosofía de la Universidad Pontificia Bolivariana, sede Medellín (Colombia). Profesor del programa de Sociología de la Universidad de Antioquia (Colombia). Correo electrónico: edinson.brand@udea.edu.co ORCID: https://orcid.org/oooo-0003-0063-5703

*** Estudiante del pregrado en Sociología de la Universidad de Antioquia (Colombia). Correo electrónico: erikaalvarezo30@gmail.com ORCID: https://orcid.org/oooo-0oo1-8787-3653
} 
**** Estudiante del pregrado en Sociología de la Universidad de Antioquia (Colombia). Correo electrónico:yuranieposso@ gmail.com ORCID: https://orcid.org/oooo-ooo2-5946-8025

**** Sociólogo de la Universidad de Antioquia (Colombia). Correo electrónico: julian.angarita3@gmail.com ORCID: https://orcid.org/oooo-0oo3-1984-8207

***** Estudiante del pregrado en Sociología de la Universidad de Antioquia (Colombia). Correo electrónico: katherine. londonoa@udea.edu.co ORCID: https://orcid.org/oooo-ooo3-4459-560o

\section{Cómo citar/How to cite}

Brand-Monsalve, Edinson Gabriel; Álvarez-Calle, Erika Yuliet; Posso-Serna, Ingrid Yuranie; Angarita-Suárez, Julián Andrés; Londoño-Arbeláez, Katherine (2021). Transformaciones más allá de la política pública de educación superior. El impacto de la formación profesional regionalizada de la Universidad de Antioquia. Revista CS, 34, 91-125. https://doi.org/10.18046/recs.i34.4226 


\section{Resumen}

El propósito de este artículo es presentar parte de los resultados de una investigación que tuvo por objetivo valorar los impactos que el acceso a la educación superior propició en la vida de un grupo de egresados del programa de Sociología, ofertado en la seccional oriente de la Universidad de Antioquia. Para alcanzar este objetivo se realizó un estudio con enfoque complementario, mediante la implementación de técnicas como la encuesta y la entrevista semiestructurada, con el fin de dar cuenta de los aspectos objetivos y subjetivos de la experiencia. El principal hallazgo evidenció que la formación profesional permitió la realización personal de los egresados como fundamento de la constitución de su proyecto de vida; ello causó transformaciones al interior de sus grupos familiares y demostró que el alcance de la política pública de educación superior va más allá de garantizar el acceso de la población a la formación profesional.

\section{PALABRAS CLAVE:}

política pública, educación superior, proyecto de vida, sociología, movilidad social

The purpose of this article is to present part of the results of an investigation that aimed to assess the impacts that access to higher education had on the lives of a group of graduates of the Sociology program, offered in the eastern branch of the Universidad de Antioquia. To achieve this, a study with a complementary approach was carried out, through the implementation of techniques such as the survey and the semi-structured interview, in order to account for the objective and subjective aspects of the experience. The main finding evidenced that professional training allowed the personal fulfillment of the graduates as the foundation of the constitution of their life project; This caused transformations within their family groups and demonstrated that the scope of public policy for higher education goes beyond guaranteeing the population's access to vocational training.

\section{KEYWORDS:}

Public Policy, Higher Education, Life Project, Sociology, Social Mobility 



\section{Introducción}

La implementación de la política pública de educación superior en Colombia se concreta a través de proyectos educativos orientados al cierre de brechas sociales, a la innovación, a la pertinencia y al aseguramiento de la calidad (Ministerio de Educación Nacional - MEN, s. f.; Departamento Nacional de Planeación - DNP, 2018), cuyos resultados pueden y deben valorarse directamente a partir de las transformaciones que se dan en las condiciones de vida de los egresados y sus entornos, en especial cuando tal implementación tiene lugar en territorios diferentes a los centros urbanos principales -educación en regiones o centros urbano-rurales (DNP, 2019)-. Tal es el caso del proyecto de regionalización de la Universidad de Antioquia - UdeA (2020), el cual tiene por objetivo implementar programas que contribuyan a principios de creación y al desarrollo de conocimiento en beneficio de la excelencia académica, la pertinencia, la interdisciplinariedad y el compromiso social (UdeA, 2017).

Un aspecto muy importante en las proyecciones de la educación profesional colombiana es la pertinencia educativa en tanto formación específica que obedece a un contexto particular, el cual conduce a que los proyectos de vida de las personas sean significados en virtud de las condiciones y posibilidades que ofrece su entorno. De ello se desprende que el sistema educativo incida “(...) en el desarrollo de habilidades, capacidades y potencialidades que fortalecen el empoderamiento del estudiante en su dimensión individual (...)" (García-Yepes, 2017: 171); trastoque las limitaciones propias de su origen social; y contribuya al cierre de las brechas sociales previas al ingreso a una institución de educación superior, muchas de las cuales se relacionan con la baja disponibilidad de recursos económicos o el cumplimiento de responsabilidades a edades tempranas que han determinado su existencia.

Por lo anterior, la implementación de la política pública de educación superior causa impactos trascendentales en la vida de las personas que transitan por la experiencia universitaria, en razón del logro de una cimentación personal a partir del papel y la experiencia que representa el egresado en la sociedad (UdeA, 2017; UdeA, 2020); además, ofrece un cúmulo de conocimientos y cumple la función de formar ciudadanos productivos con oportunidades de ampliar competencias (MEN, s. f.) que les representen herramientas para el trabajo y el desarrollo humano (DNP, s.f.).

El papel que cumplen los profesionales en la sociedad está anclado a las transformaciones generadas en las historias de vida y en la configuración de sus motivaciones no solo frente al ingreso a la educación (Yaschine, 2015), sino respecto a los cambios generacionales que este acceso causa (Rodríguez, 2016); a la reconstrucción y resignificación de los proyectos de vida (García-Yepes, 2017; López-Cárdenas; Mella-Luna; Cáceres-Valenzuela, 2018); a la edificación y satisfacción con la consolidación de 
una trayectoria profesional (Vázquez-González; Díaz-Pérez; Pérez-Damian, 2015), y a la constitución de una estabilidad que se complementa con el hecho de ser la educación el factor que da apertura a la movilidad social, o ayuda a resguardar posiciones sociales alcanzadas (Navarro-Leal; Sembler-Reyes, 2018).

La experiencia formativa, que inicia desde la escuela, contribuye a la construcción de un proyecto de vida enmarcado en múltiples estrategias y acciones implementadas para lograr objetivos formativos a partir de la autonomía y la proactividad del individuo (García-Yepes, 2017), los cuales, en etapas superiores-nivel universitario-, conllevan cambios en sus condiciones de vida. Esto por cuanto, como lo establecen González, Díez-Echavarría, Zapa y Eusse (2019), cuando un programa es pertinente, sus egresados permanecen empleados y generan mejoras en tales condiciones; en otras palabras, existe una relación entre pertinencia, empleabilidad y mejoramiento de las condiciones de vida de las personas que se titulan.

En estos procesos de configuración, en los cuales los estudiantes desean y tienen la posibilidad de cambiar o hacer perdurar las características de sus realidades, el accionar de la institucionalidad constituye "(...) un elemento muy importante en la construcción del sentido de los estudios y de la identidad" (Guzmán-Gómez, 2017: 75). Lo anterior porque esta última se configura a través de las opciones de vida, las cuales aparecen frente a los educandos, potencializan y organizan lo que se quiere "ser y hacer" en relación con sus proyectos de vida, y generan virajes en las formas de pensar y percibir el mundo, así como en las percepciones que se ven relacionadas muchas veces con el origen social. A partir de ello se establecen proyecciones sobre lo que se quiere lograr o, por el contrario, se genera un factor de incertidumbre frente al futuro (Gómez-Urrutia; Royo-Urrizola, 2015).

Como producto de la experiencia educativa emerge una diversidad de motivaciones que permiten otro tipo de accionar, tanto en los estudiantes como en los egresados. Estas van desde continuar con los estudios y realizar actividades de actualización en conocimientos (González et al., 2019) hasta posponer la formación y la independencia familiar, debido a que se busca primero asegurar todo lo que atañe a la sostenibilidad y la estabilidad económica (Díaz; Galán, 2015).

En este marco, se reconoce que muchas de las investigaciones sobre el desarrollo e impacto de los programas universitarios tienen un acercamiento a la vida y logros de los estudiantes y egresados: hacen más énfasis en el vínculo con el mercado laboral y en la evaluación de la eficacia de los programas (Fernández-Esquinas, 2019; Facchini, 2019; Gutiérrez; Mondragón; Santacruz, 2019), en las que se indica -en el caso del pregrado en Sociología- que los planes de estudio estructurados por las universidades no pueden satisfacer en su totalidad las demandas teóricas y metodológicas requeridas por los aprendices, ni la necesidad de forjar cambios de 
modo permanente, en función del mercado de trabajo (Diez, 2017); o bien, como lo mencionan Malagón-Plata, Rodríguez-Rodríguez y Machado-Vega (2019), las instituciones de educación superior ostentan inflexibilidad sobre las culturas académicas y administrativas, las cuales hacen que sea difícil la ejecución de las políticas. No obstante, en estos trabajos no se reflejan de formas amplia y clara las transformaciones generadas por la educación superior en la experiencia de las personas en relación con su motivación por la formación universitaria.

En Latinoamérica, el abordaje de la experiencia de los profesionales en sociología se ha centrado en, de un lado, valorar la pertinencia de la educación recibida al ingresar al mercado laboral (Fernández-Esquinas, 2019); y de otro, en identificar condiciones profesionales y actividades más recurrentes, vinculadas a la primera experiencia de trabajo (Beltrán; Berrío-Gil, 2016; Diez, 2017). También se encuentran estudios que abordan las percepciones de los graduados sobre el programa (Beltrán; Torres-Ortiz, 2016), en los cuales se hace énfasis en las limitaciones encontradas por los profesionales al momento de ingresar al mercado laboral; encuentran que el trabajo del sociólogo, fuera del campo académico, puede ser visto como una carrera menor, al punto que se la asemeja a ser técnica, o a estar sometida al mercado (Baltar; Siqueira-Baltar, 2017). En este marco, es necesario ir más allá de estos asuntos y reconocer el papel que el acceso a la educación superior tiene en la configuración del proyecto de vida de las personas que estudian sociología y de sus entornos inmediatos, en especial el familiar.

Con lo expuesto, el objetivo del estudio presentado aquí fue comprender las trasformaciones que el acceso a la educación superior causó en la experiencia de vida de un grupo de egresados del programa de Sociología, el cual es ofertado en la seccional de El Carmen de Viboral de la Universidad de Antioquia. Se identificaron las características de movilidad social y ocupacional respecto a sus condiciones de origen familiar, para lo cual se partió del supuesto de que el cumplimiento de la política pública de educación superior va más allá de su alcance técnico, pues no se trata solo del acceso a la titulación, sino de la consideración de que la experiencia educativa transforma la vida de las personas.

El interés por abordar la vivencia de los egresados de Sociología en esta sede obedece al propósito institucional -en el marco del proceso de autoevaluación del programa- por reconocer los alcances de la formación en esta disciplina fuera de la ciudad capital del Departamento de Antioquia. Con esta pretensión se busca contribuir al debate sobre la importancia de dar continuidad a la oferta académica de dicha carrera para este territorio particular al considerar que, en primer lugar, y pese a que el espacio nombrado fue el primero en que se ofreció el programa -en el marco de la política de descentralización de la educación pública consignada en el 
proyecto de regionalización de la Universidad de Antioquia- (UdeA, 2O2O), no se cuenta con ningún estudio que ofrezca elementos al respecto.

En segundo lugar, el Oriente Antioqueño ha tenido un gran crecimiento urbano en los últimos años, debido a la cercanía con el Valle de Aburrá (centro urbano más próximo), el desplazamiento y ampliación de industrias en esta zona, el acceso y la comunicación de vías principales del Departamento y el mejoramiento de redes viales, entre otros aspectos. Todo ello está acompañado por procesos de desarrollo social y fortalecimiento de las organizaciones sociales y de base, lo cual constituye múltiples escenarios de interés sociológico que demandan la presencia de profesionales de la disciplina.

En tercer lugar, y según el periódico Alma Mater (2003) de la institución estudiada, por año, solo en El Carmen de Viboral -uno de los once municipios del territorio conocido como el Oriente Antioqueño cercano- egresaban 500 estudiantes para la época, de los cuales solo el 7 \% accedían a la educación superior, situación que redundaba en un gran porcentaje de jóvenes desocupados. En este marco, la sede universitaria llegó para propiciar oportunidades de educación en la población juvenil de la región y facilitar el acceso a estudiantes rurales que, por motivos económicos, no pueden abandonar su territorio en busca de oportunidades educativas. Con esta nueva opción de educación superior se da una posibilidad de construir procesos sociales desde su propio territorio, factor que posibilita la no migración por falta de acceso educativo.

Los resultados presentes a continuación dan cuenta de este escenario de oportunidad, en el cual se encuentra, como principal hallazgo, que la formación profesional permitió la realización personal de los egresados como fundamento de la constitución de su proyecto de vida y generó transformaciones al interior de sus grupos familiares, lo cual demuestra que el alcance de la política pública de educación superior va más allá de garantizar el acceso de la población a la formación profesional al posibilitar no solo la titulación, sino la generación de espacios académicos y sociales para la acumulación de diferente tipos de capitales que amplían y modifican la trayectoria de vida de los educandos y de sus redes personales, familiares y de empleabilidad más cercanas, lo que a su vez se traduce en cambios en la estructura social de los territorios.

Para efectos de la exposición de los resultados que sustentan estos hallazgos se presentan tres argumentos, precedidos por una conceptualización basada en los conceptos de motivación y proyecto de vida, y de la exposición detallada de la metodología e instrumentos utilizados. En primer lugar, se hablará del logro personal como fundamento de lo motivacional para profesionalizarse; se mostrará el contraste entre las condiciones educativas de origen familiar frente al principal aspecto que motivó el acceso a la educación superior para los casos abordados. En 
segundo lugar, se desarrollará el argumento de la acumulación de capitales como eje de la movilidad ocupacional, dentro de lo cual se expondrá el papel de los capitales cultural y social para lograr el ejercicio profesional. En tercer lugar, se presentará el logro educativo como un factor transformador de los vínculos familiares y personales más cercanos de la población abordada. Y a modo de cierre, se desarrollará un apartado de conclusiones en el que se presentarán los aspectos más relevantes de los resultados obtenidos para este estudio.

\section{La motivación y el proyecto de vida: conceptos fundamentales para la comprensión de la movilidad social}

Para efectos del presente estudio, se abordaron los conceptos de motivación y proyecto de vida como bases para comprender el fenómeno de movilidad social en la experiencia de vida que tuvo la población abordada en virtud del acceso a la educación superior; dentro de esto, la motivación por el logro educativo jugó un papel determinante para el acceso y la culminación de la formación profesional, toda vez que los deseos se tradujeron en acciones realizadas para lograr transformaciones en sus condiciones de vida (Giddens, 2006: 43-44). Se hace importante dejar claro que en las modificaciones del entorno -familiar, social, cultural e histórico- es determinante y, por tanto, de obligatorio abordaje (Lomelí-Parga; López-Padilla; Valenzuela-González, 2016), el individuo, pues ejerce acciones para la construcción y realización de su proyecto de vida.

En este sentido, las acciones concretan las motivaciones del agente. Por tanto, el acceso a la educación superior constituye una acción que denota las motivaciones de los individuos abordados para configurar un proyecto de vida profesional que redunde en modificaciones a las condiciones de origen en las cuales tuvo génesis su trayectoria de vida, y en las que se reconocen las situaciones estructurales presentadas como habilitantes o limitantes para alcanzar las metas de cada plan de vida, pues la estructura social cumple esta doble función: genera restricciones y brinda oportunidades para que el agente sea capaz de emprender accionares (Guzmán-Bracho, 2019) a traducirse en transformaciones como resultado del logro de objetivos trazados de tal forma que se alcance un escenario deseado, toda vez que "(...) ser un agente es ser capaz de desplegar (repetidamente, en el fluir de la vida diaria) un espectro de poderes causales, incluido el poder de influir sobre el desplegado de otros" (Giddens, 2006: 51).

La capacidad de desplegar acciones basadas en un poder causal, esto es, orientado a generar un resultado deseado, está relacionada, desde la perspectiva aquí adoptada, con los capitales acumulados por el agente (Bonnewitz, 2006). Por ello deben 
considerarse la cantidad y la calidad de los mismos, pues la segunda se entiende en relación con las ventajas que le provee al individuo.

La universidad cumple un papel fundamental para el agente en la gestión de diferentes tipos de capitales, en tanto le permite potenciar los acumulados durante la trayectoria de vida previa a la experiencia universitaria, así como adquirir nuevos en el proceso formativo, principalmente, capital cultural en sus tres formas o estados (Bourdieu, 2001). En primer lugar, como capital cultural incorporado, es decir, aquel que presupone un proceso de interiorización de conocimientos en un tiempo prolongado, pues implica un periodo de enseñanza y aprendizaje y constituye una posesión que se convierte en parte de la persona, a saber, prácticas sociales ordenadas en un espacio y tiempo (Bonnewitz, 2006). En segundo lugar, como capital cultural objetivado, que corresponde al conocimiento que se convierte en una forma materialmente transferible a través de libros, escritos, cuadernos, diccionarios, entre otros. Y, en tercer lugar, como capital cultural institucionalizado, el cual es una forma de objetivación del capital cultural incorporado como títulos. Es importante tener presente que la acumulación de estos capitales no ocurre porque estén distribuidos de forma igualitaria entre todos los individuos, sino que tal acumulación es resultado de disputas intelectuales sobre teorías y sus críticas, en lo cual intervienen múltiples factores.

Junto con el desarrollo de habilidades cognitivas, la universidad permite generar prácticas de socialización conducentes al establecimiento de conexiones sociales, las cuales se tornan estables y duraderas en función de las interacciones que forman parte del proceso de formación y representan acumulación de capital social, el cual “(...) constituye una inversión en relaciones sociales como fuentes de recursos a la que los individuos pueden acceder y movilizar" (Lin et al., como se citó en Marqués-Perales; Gil-Hernández, 2015:7) en virtud de pertenecer a un grupo privilegiado, y que se pueden transformar en posteriores beneficios.

En el marco de la motivación y de la acumulación de capitales, el proyecto de vida se desarrolla en virtud de un “(...) proceso que estructura tanto expectativas vitales como las estrategias para lograr dichos objetivos en el marco de un contexto social" (García-Yepes, 2017: 154). Este permite transformar las condiciones de origen al ampliar las posibilidades de interacción en otros espacios y grupos que el proceso educativo, en instituciones de educación superior, provee; a la vez, cualifica las habilidades y destrezas utilizadas por el agente para desempeñarse en el campo profesional, el cual incluye el mercado laboral. 


\section{Metodología}

Para el cumplimiento del objetivo del estudio se empleó una metodología integrada, entendida también como complementaria, la cual corresponde a "(...) una estrategia de integración multimétodo utilizada en una investigación en la cual teniendo solo un objeto de estudio se aplican ambos enfoques, el cualitativo y el cuantitativo, obteniendo dos imágenes, una proveniente de cada enfoque" (Blanco; Pirela, 2016:106).

Desde esta concepción de complementariedad, y de acuerdo con Veliz-Rojas y Bianchetti-Saavedra (2016), se abordó el objeto de estudio desde la perspectiva objetiva y subjetiva de los participantes al buscar su caracterización, así como el reconocimiento del sentido implícito en él. Para ello, en primera instancia, se hizo uso de la técnica de la encuesta (Vieytes, 2004), cuyo diseño fue producto de la operacionalización de los conceptos anteriormente expuestos; a partir de ello se construyeron 58 variables de tipo demográfico, socioeconómico, cultural, de empleabilidad y de redes, agrupadas en tres momentos de la trayectoria de vida de los egresados: las condiciones de origen social, el proceso de formación profesional y el ejercicio de la profesión. En este último momento se incluyeron, además de las condiciones de participación en el mercado laboral, el uso de redes para el acceso a puestos de trabajo de acuerdo con el modelo de Granovetter (1995), en el que la aplicación a tales puestos se presenta a través de vínculos fuertes o débiles (Granovetter, 1973).

En provecho de las ventajas de la integración de métodos (Puentes-Borges; Puentes-Bencomo; Puentes-Bencomo, 2017; Alzás-García; Casa-García, 2017; Sánchez, 2015) se diseñó la guía de entrevista semiestructurada (Trindade, 2016), a partir de los resultados de la encuesta, para profundizar sobre las razones por las cuales los participantes del estudio decidieron orientarse hacia la formación profesional y cómo esta transformó su vida y la de sus familiares y personas cercanas. La guía se diseñó sobre la base de los tres momentos de la encuesta; y el análisis de la información se realizó de acuerdo con un proceso de codificación cerrado, en el cual se utilizó la técnica de redes semánticas a partir de tres categorías principales: logro personal, logro educativo y acumulación de capitales. En función de estas tres categorías se profundizó en la información relacionada con las características de las motivaciones que, para los egresados, fueron determinantes en la decisión de acceder a la educación superior y en la forma como la experiencia educativa marcó sus trayectorias familiares, educativas y profesionales. De esta manera, se ubicaron los roles que a tal respecto tuvieron la familia, los amigos, las instituciones educativas y los docentes, entre otros grupos relevantes, para identificar el impacto de la educación superior en el egresado, más allá del cumplimiento de la política pública en la región. 
La población abordada correspondió a 28 egresados de los 34 registrados en la base de datos de la seccional, esto es, el $82 \%$ del total de la población. El criterio de inclusión que se aplicó en general fue la disponibilidad para la participación en la investigación, de acuerdo con el contacto efectivo con los datos disponibles y la realización de la técnica de bola de nieve. En términos de distribución de la población, se abordó a 13 hombres y 15 mujeres, casi la totalidad con edades entre 26 y 37 años, habitantes de los municipios de El Carmen de Viboral, la Ceja y Rionegro en la mayoría de los casos, y con más de dos años de egreso del programa.

La aplicación de los instrumentos se hizo por método directo (cara a cara) en el lugar de trabajo o residencia de cada una de las personas contactadas. Tuvo una duración promedio de 45 minutos para la encuesta, y de 60 a 90 para la entrevista. La aplicación se acompañó del consentimiento informado, en cumplimiento de lo establecido en el Comité de Bioética de la Facultad de Medicina de la Universidad de Antioquia, conforme a la Declaración de Helsinki 2002, la Resolución o08430 de 1993 del Ministerio de Salud y Protección social, normas éticas internacionales para la investigación en humanos y el Decreto 2378 de 2008. El análisis de datos se adelantó con asistencia de las aplicaciones informáticas SPSS y Atlas.Ti; se aplicaron procedimientos de estadística descriptiva para el caso de los datos cuantitativos y de análisis categorial y construcción de redes semánticas para los cualitativos, lo cual permitió generar una integración de la investigación en el nivel técnico, es decir, una triangulación de datos (Aguilar-Gaviria; Barroso-Osuna, 2015; Veliz-Rojas; Bianchetti-Saavedra, 2016).

\section{Resultados}

\section{Logro personal como fundamento de la motivación para profesionalizarse}

Para la mayoría de los egresados abordados en este estudio, el acceso a la educación superior fue una circunstancia novedosa en su trayectoria de vida. En 26 de los 28 casos analizados, esta fue la primera experiencia familiar en educación superior caracterizada por un débil encauzamiento educativo desde la familia, a causa de un nivel formativo bajo en las generaciones precedentes, esto es, abuelos y padres: de forma mayoritaria, los primeros cursaron solo la educación primaria o, en su defecto, ningún proceso formativo de carácter formal, y los segundos, únicamente la primaria o secundaria. Adicionalmente, se registra poca dedicación a actividades intelectuales en estas dos generaciones; en menos de la mitad de los casos (39,3\%) se reconoció la existencia regular de alguna práctica constante de este tipo en los 
padres: por ejemplo, menos de la mitad se dedica a la lectura (figura 1), situación que se explica por cuanto la mayor parte de ellos desempeña oficios no cualificados, al tiempo que están asentados en territorios donde no contaron con acceso a la educación superior, con lo que esta se torna en un ámbito poco o nada significativo en la consolidación de sus proyectos de vida. No obstante, la situación de los padres fue un elemento que llevó a los egresados a buscar la calificación a través de la formación universitaria, así como al cultivo de diferentes prácticas intelectuales-la dedicación a la lectura, por ejemplo-.

\begin{tabular}{|l|l|}
\hline FIGURA 1 & $\begin{array}{l}\text { Prácticas intelectuales en la familia del egresado antes de su ingreso } \\
\text { a la universidad }\end{array}$ \\
\hline
\end{tabular}
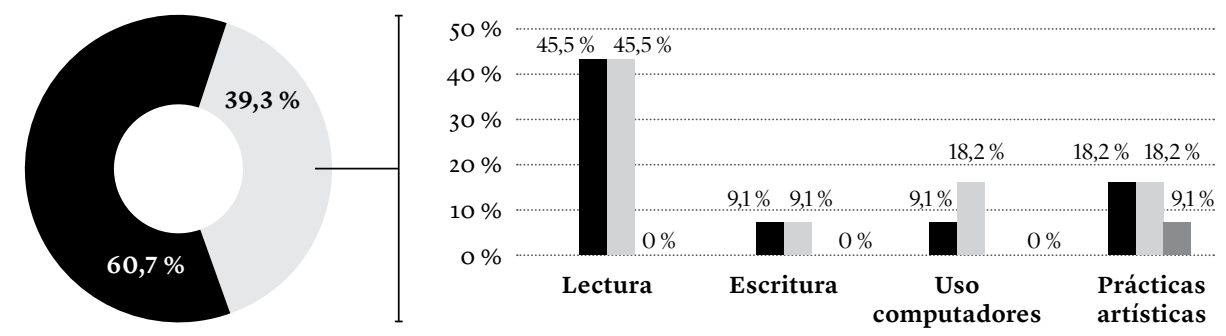

\begin{tabular}{|c|c|c|c|c|}
\hline No & Sí & a Padre & Madre & Abuelo \\
\hline
\end{tabular}

Aunque gran parte de los padres no tuvieran un papel activo en el encauzamiento educativo de los hijos, el 71,4\% sí tenían un interés en que estos lograran profesionalizarse. La expectativa por la profesionalización de los hijos estaba fundamentada en el deseo de que tuvieran mejores condiciones de vida, en especial en lo tocante a lo material, pues tal expectación estuvo asociada principalmente al progreso económico, seguido del intelectual (figura 3). Con lo segundo -hallado en las entrevistas- se guardaba la intención de que los hijos tuvieran la oportunidad de ocuparse en un trabajo cualificado, el cual les posibilitara, además de mejores ingresos, una actividad laboral intelectual.

En contraste, y en este contexto familiar, el cual se caracteriza por un bajo nivel educativo de padres y abuelos, y una alta expectativa sobre el logro de educación superior para los hijos, la búsqueda de los egresados por la formación universitaria se orientó, ante todo, hacia alcanzar su realización personal, seguido de modificar 
sus condiciones de vida. Según sus discursos, lo más importante para ellos era consolidar un proyecto de vida basado en el logro educativo, con el fin de potencializar su sentido de pertenencia con lo social, al entender la formación sociológica desde la perspectiva del aporte a las personas y al entorno. Esta situación pudo evidenciarse a partir de los datos de la encuesta: el aspecto con mayor calificación, respecto a la motivación de estudiar una carrera profesional, fue la realización personal.

Al relacionar gráficamente la calificación de este aspecto en una escala numérica de o a 5-donde 5 representaba el mayor valor de relevancia para estudiar una carreracon el nivel educativo de los padres y abuelos para cada uno de los egresados-donde o corresponde a ningún grado formativo y 5 al nivel de posgrado-, se observa que la búsqueda por la realización personal no estuvo asociada a la condición académica e intelectual de la generación precedente, como se muestra en la figura 2. Es decir, se trata del deseo por lograr transformar la condición de capital cultural de origen, lo cual se configura como principal motivación. Esta situación fue posible, para los egresados, a partir de las condiciones habilitantes del entorno: esto es, la existencia de una oferta educativa accesible que viabilizó su ingreso a la educación superior, \begin{tabular}{l|l} 
FIGURA 2 & $\begin{array}{l}\text { Motivación para profesionalizarse del egresado frente al nivel educativo } \\
\text { familiar }\end{array}$
\end{tabular}

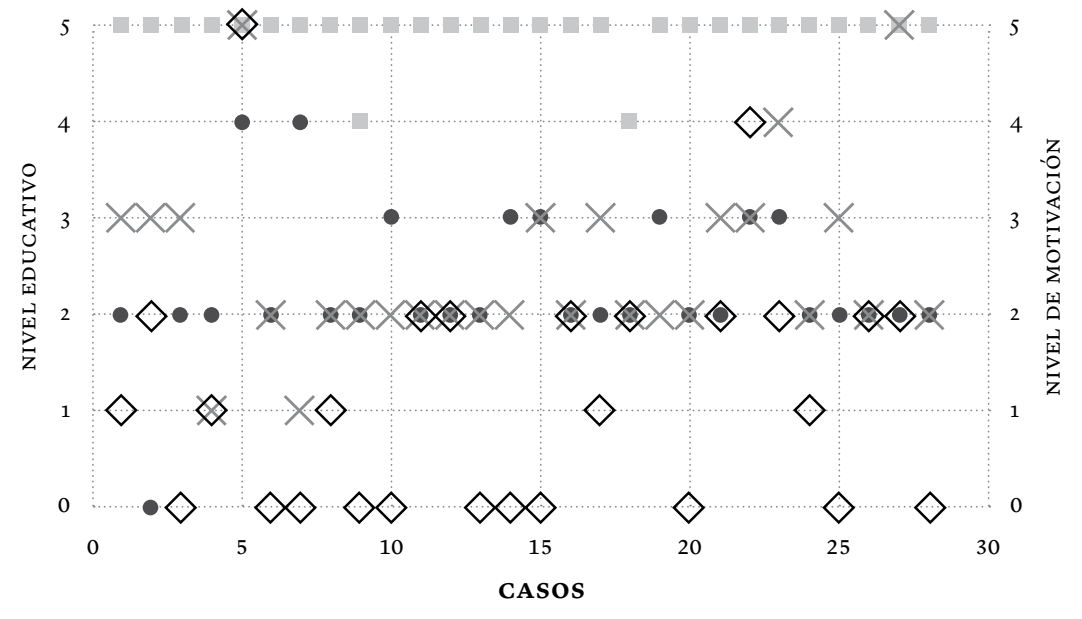

Nivel educativo del padre

$X$ Nivel educativo de la madre

$\diamond$ Nivel educativo del abuelo

Motivación por la realización personal.

Para una mejor comprensión se deben observar los cuatro valores de cada caso. 
pues en casi la totalidad de los casos esto fue determinante para estudiar y obtener la titulación profesional -en otras palabras, cumplir con la aspiración de realizarse personalmente a través de la formación universitaria-.

Se debe resaltar el hecho de que, en el acceso a la educación, el papel de la familia no se redujo solo a la existencia de una expectativa educativa sobre los hijos, sino al principal apoyo para que estos lograran culminar los estudios: en la mayoría de los casos (85,7\% del total), proporcionaron el pilar emocional para mantenerlos motivados en alcanzar la meta de titularse. En virtud de ello, los egresados pudieron superar las dificultades que se presentaron durante su proceso formativo en la universidad, pues como lo manifestaron en las entrevistas, por ser los primeros en vivir dicha experiencia, sentían la presión por lograr y mantener un buen desempeño académico, para lo cual necesitaron sentir el respaldo emocional permanente de sus madres y padres para, de esta forma, mantener su motivación por el proceso emprendido.

Aquí, el papel de abuelos, padres, hermanos y parejas, entre otros, fue determinante para que los titulados no suspendieran o abandonaran los estudios y lograran el propósito de la política pública de educación superior, es decir, cumplieran con el acceso a un cupo en una institución universitaria; y que, más allá de esto y de la política en sí, vieran las posibilidades -diferenciadas- ofrecidas por este tipo de educación, y en esa medida, gestionaran acciones que les llevaran no solo a estudiar y obtener conocimientos, sino a ponerlos en práctica, todo ello conducente a lograr una vida diferente, principalmente frente al logro de estabilidad ocupacional. De igual forma, la familia representa un apoyo económico para lograr sostenibilidad al cubrir los gastos básicos de la estadía en la universidad, lo cual se registró en el 75 $\%$ de los casos. Todo lo anterior evidencia que la educación de uno de los miembros se tornó en un proyecto colectivo de la familia.

En el proceso de concretar la motivación por estudiar en acciones efectivas y transformarlas en un logro en el proyecto de vida propio y de los allegados, ante la ausencia de una experiencia educativa en la mayoría de los grupos familiares, algunos vínculos externos a la familia tuvieron un papel fundamental. Sobre esto se observa que los profesores de la básica secundaria y los amigos fueron los dos grupos de mayor influencia en la decisión de estudiar una carrera, con valores promedios de 2,86 y 2,71 respectivamente, en una escala de valoración de la influencia medida de 1 a 5 . Estos representan una experiencia valiosa en la medida en que el escenario intelectual era muy reducido y no se contaba con muchas vivencias cercanas y exitosas en la educación superior.

Es importante mencionar que 71,4 \% de los egresados afirmaron haber recibido consejos de uno o más profesores del colegio, lo cual fortaleció el deseo por acceder a la educación superior $y$, en algunos casos, fue un factor determinante, pues los 
motivaron a emprender un proyecto que hasta el momento era desconocido. Tanto los datos de la encuesta como los de la entrevista evidencian que el papel cumplido por los docentes en este nivel educativo constituye un aporte significativo para la efectividad de la política pública de educación superior, toda vez que propicia en los estudiantes la motivación para estudiar una carrera profesional y aprovechar la oferta educativa en la región.

La influencia de estos grupos permitió consolidar una expectativa de realización personal a través del estudio, pues convirtió el logro educativo en la base del proyecto de vida de los ahora egresados, orientado principalmente, como se mencionó, por el deseo de progresar intelectualmente, es decir, por poseer conocimientos cualificados a través de la formación y obtener un progreso económico. Es posible contrastar esta situación con las expectativas de los padres, para quienes este último aspecto sí tenía más relevancia en el logro educativo de sus hijos, tal como se muestra en la figura 3: allí donde se presenta el promedio de calificación de los 28 casos -en una escala de o a 1- sobre los aspectos que tuvieron mayor importancia para los egresados y, en su opinión, para sus padres al tomar la decisión de acceder a la educación superior -donde 1 corresponde a la mayor importancia-.

\section{a la educación superior}

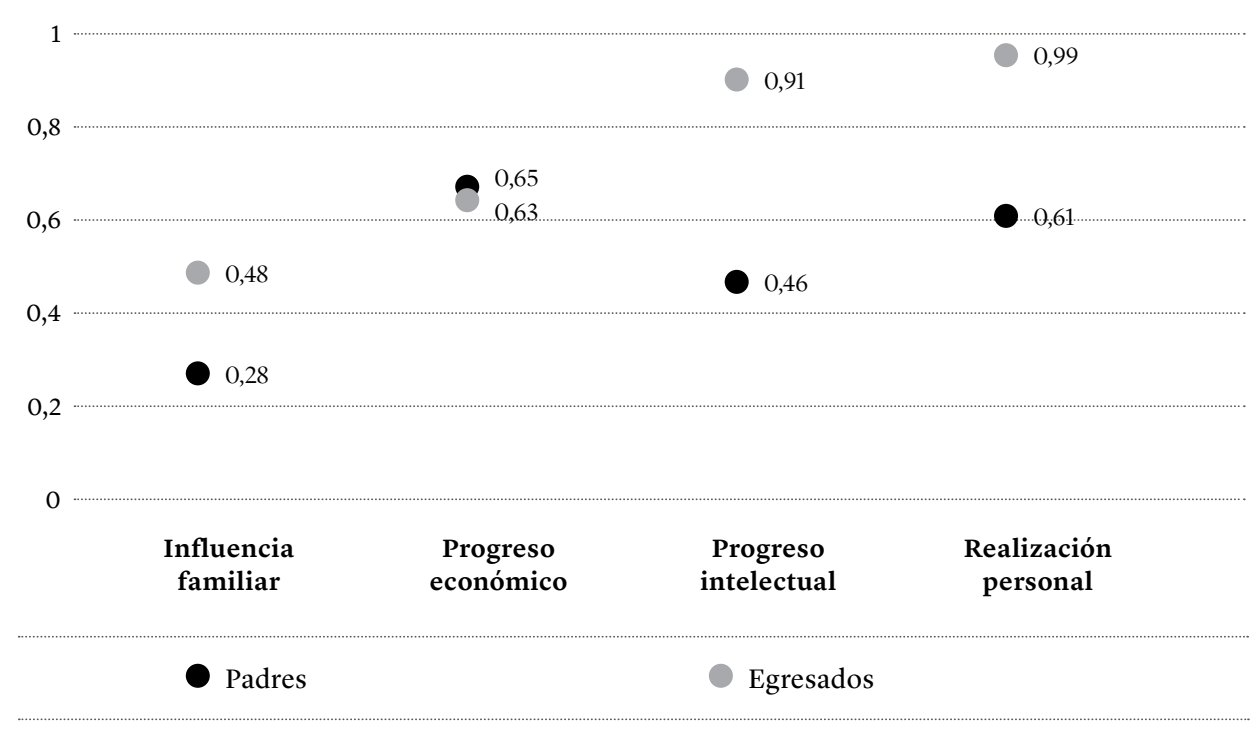


En las entrevistas, los egresados manifestaron que la presencia de la universidad fue un factor determinante para que sus expectativas personales y familiares se convirtieran en importantes logros en sus vidas pues, para la mayoría de ellos, la cercanía y el bajo costo se convirtieron en aspectos definitivos para lograr el acceso a la educación superior (con una calificación promedio de 4,64 y 4,54, respectivamente, en una escala de valoración de aspectos influyentes para estudiar, medida de 1 a 5). Para ellos era impensable desplazarse al área metropolitana o acceder a una universidad privada con presencia en el territorio cercano, por los elevados costos que implica. Por ello, el hecho de entrar a la universidad fue, de sí, un gran logro que generó gran satisfacción personal. De esta forma, en el sentir de los egresados, la oferta de la Universidad de Antioquia no solo constituyó la ventana de oportunidad para ellos (y para muchos otros) como parte del sistema de educación superior que garantiza el cumplimiento de la política pública educativa para este nivel, sino que se tornó en la posibilidad de transformar sus vidas, al permitirles hacer realidad sus expectativas y motivaciones.

La universidad llegó con una amplia oferta de pregrados -diversidad de ingenierías, Trabajo Social y Psicología, entre otros-. Esta situación enfrentó a los egresados a la decisión de escoger uno entre tantos programas. De acuerdo con las entrevistas, se identifican tres situaciones en la elección de la disciplina de la Sociología. En primer lugar se encuentran las personas que no tenían claridad ni conocimientos previos y definidos sobre la profesión a elegir, es decir, no contaban con una vocación definida, y optaron por aplicar a la oferta disponible para esta sede de la Universidad en el momento particular; en segundo lugar, aquellos que reconocen haber estado cerca de la vocación social desde temprana edad -si bien no directamente a un pregrado específico, con inclinación a la comprensión de las realidades sociales-; y en tercer lugar, los casos de quienes tenían plena claridad de optar por la Sociología como profesión, situación vinculada a experiencias propias. Así, se encontraron situaciones significativas en las cuales muchos de los individuos, a medida que avanzaban en la carrera y conocían todo lo que les aportaba en los planos metodológico y teórico, se motivaban a continuar, mientras que otros fortalecían su pasión por el ámbito social.

El paso por la Universidad abrió las puertas de un mundo desconocido para los egresados. Allí adquirieron nuevas experiencias, conocimientos y relaciones sociales, entre otros aspectos que los enorgullecen y los llenan de satisfacción. Hoy, desde su sentir, se consideran personas exitosas en diferentes ámbitos, sin que ello signifique sentirse plenos; no obstante, reconocen los logros obtenidos que han transformado sus vidas y la de sus familias. En promedio, el aspecto en el que refieren haber alcanzado mayor éxito -haciendo una valoración en una escala de o 
a 1, donde 1 corresponde al mayor nivel de éxito concierne a la realización personal que, desde un principio-, fue la mayor motivación en su plan de vida en relación con la educación, seguido del desarrollo intelectual (figura 4); sin embargo, de acuerdo con la calificación otorgada en la escala, el aspecto en el cual aún consideran tener mucho pendiente por lograr corresponde al progreso económico.

\section{FIGURA 4 $\quad$ Nivel de satisfacción de los egresados en diferentes ámbitos}

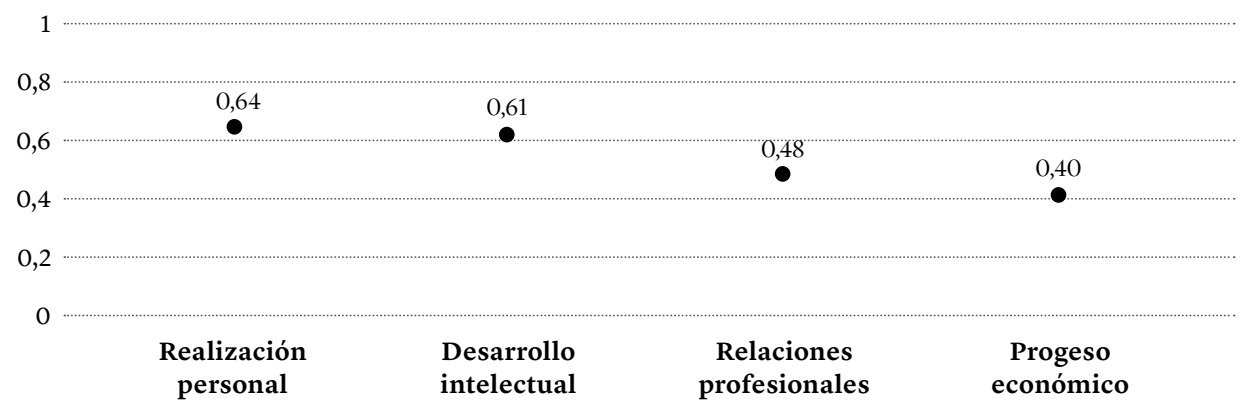

Fuente: elaboración propia.

Esta realización personal e intelectual se acompaña de haber logrado vincularse al mercado laboral. Este aspecto les brinda la posibilidad de compartir sus saberes y ofrecer estrategias relevantes, las cuales contribuyen a la labor social que desempeñan. Al menos la mitad de los egresados expresan gratitud por lograr integrarse a empleos relacionados con la formación recibida, situación que les ha traído satisfacción económica y profesional. En correspondencia, el factor económico comienza a tener fuerza e importancia y deja de estar en un segundo plano en las vidas actuales de los egresados, en gran parte porque saben que de ello depende la continuidad educativa. De esta manera, expresan la importancia de ganar experiencia laboral y cualificarse más para lograr un mayor ingreso, sobre el cual anotan que han encontrado grandes diferencias con otras carreras en el campo laboral.

Es importante apuntar que algunos profesionales no se han podido desempeñar como sociólogos. Esta situación les causa insatisfacción, aunque se sienten realizados en lo personal por el logro educativo porque les ayudó a comprender y mejorar las diversas realidades encontradas en la cotidianidad, y tomar parte en conversaciones académicas, políticas y sociales con herramientas teóricas y prácticas útiles, obtenidas en su formación para su construcción como personas críticas. 
Con base en lo planteado, se reconoce la existencia de otros elementos más allá de los dispuestos en la política pública educativa, que muestran otra cara de su implementación: existen circunstancias que no solo dan cuenta del desempeño de una persona mientras cursa sus estudios, sino después de concluirlos, periodo en que se desarrolla un sinnúmero de experiencias significativas (diferentes tipos de trabajo, discusiones interdisciplinares, nuevos relacionamientos, entre otros asuntos), así como numerosas dificultades que configuran la experiencia de vida de la persona.

\section{La acumulación de capitales como eje de la movilidad ocupacional}

El título profesional constituye un elemento institucional que lleva a la materialización de las acciones realizadas por los egresados para alcanzar las metas que se trazaron en función de su proyecto de vida, motivado por el logro educativo. Esta titulación representa una trayectoria académica en la que se acumularon diferentes capitales, traducidos principalmente en habilidades cognitivas, así como en nuevas relaciones de conocimiento y reconocimiento propio y de otras personas. Dicho cúmulo les representa recursos que incrementan su capacidad de negociación en el mercado laboral, es decir, medios para acceder y mantenerse en una posición dentro del mismo.

Uno de los cambios más notables para los egresados se da en función de la ocupación de sus padres y abuelos: en el $75 \%$ de los casos, estos desempeñan trabajos no cualificados o manuales con bajo reconocimiento económico, relacionados especialmente con labores del campo, oficios de construcción y proyectos independientes. Se presenta en este sentido una situación de poca variación, dado que permanecen en los mismos puestos de trabajo durante toda su vida en pro de salvaguardar la estabilidad, pese a no tener buena remuneración. Sumado a esto, en la mitad de los casos las madres han cumplido labores de cuidado y del hogar. Frente a estas circunstancias, los egresados fueron expresos en sus relatos respecto al deseo por transformarlas a partir de convertirse en profesionales para no reproducir el mismo ciclo y, de esta manera, lograr mejores empleos y remuneraciones -y para el caso de las mujeres, abrirse a un mundo diferente al de permanecer en sus casas y cumplir con funciones del hogar-.

Frente a la empleabilidad, es significativo encontrar que el 67,2 \% de los egresados trabajaron durante todo o gran parte del tiempo en que fueron estudiantes universitarios, y 1 de cada 4 laboró en un empleo relacionado con la carrera. De aquí se desprenden dos asuntos importantes expresados por los graduados, sin desconocer los retos afrontados por el estudiante trabajador para mantener un buen rendimiento académico. En primer lugar, la familiarización con el mundo laboral 
antes de la graduación representó una ventaja para acceder a un empleo como profesional, pues la experiencia respecto al cumplimiento de metas, la capacidad para trabajar en equipo y la negociación, entre otros aspectos, fueron aprovechadas al enfrentarse a escenarios de competencia por puestos de trabajo. En segundo lugar, quienes se vincularon en cargos relacionados con la formación sociológica $-24 \%$ de los casos- tuvieron una ventaja adicional frente a los demás estudiantes, en la medida en que ponían en práctica y reforzaban los contenidos desarrollados a lo largo del programa, y adquirían y fortalecían relaciones que luego les representaron mejores oportunidades para la consecución de un empleo.

No obstante, se encontró que 53,6\% de los graduados-sumados los porcentajes de casos correspondientes a la cantidad de meses o, 1 y 2-lograron conseguir su primer empleo en menos de 2 meses, y el $75 \%$ en un tiempo máximo de 6 meses -sumados los porcentajes de la cantidad de meses o a 6- (figura 5). Esto es significativo para el campo de acción de la Sociología, dado el bajo reconocimiento a la labor y el perfil de esta profesión según la experiencia de los egresados. Así mismo, es relevante valorar la formación sociológica respecto al requerimiento de la política pública de educación superior en lo tocante al desarrollo de herramientas para el trabajo, las cuales permiten el logro ocupacional por parte de los sociólogos. Situación similar se tiene al indagar por el último o actual empleo: 57,1 \% logró ocuparse en menos de 2 meses y el $78,5 \%$ tardó 6 meses como máximo, lo que da cuenta de una dinámica significativa en la movilidad ocupacional de esta población.

Aunque algunos profesionales cuentan que les ha costado mantenerse empleados en oficios relacionados con su carrera, la mayoría reconoce haber podido ejercer la mayor cantidad de tiempo desde la obtención del grado, aunque con periodos sin ocupación: de 1 a 6 meses para el $46,5 \%$ de los casos, y hasta 2 o más años para el $21,5 \%$. Esta situación está ligada a las condiciones ofrecidas por los empleadores y, en sí, a las dinámicas del mercado laboral en la región, donde la mayoría de los contratos se encuentran relacionados con proyectos de tiempos específicos y limitados.

En esta situación de acceso al primer y a los demás empleos resulta fundamental considerar el papel que cumple el capital social acumulado (Bourdieu, 2001) durante el pregrado y después de él, por cuanto el acceso a la educación superior permitió adquirir conocimientos, habilidades y destrezas en diferentes grados, así como nuevas relaciones que luego se tradujeron en vínculos fuertes, los cuales ayudaron para el ingreso y cambio de ocupación, es decir, en una tarea de sociabilidad (Bonnewitz, 2006). Por esta razón, compañeros o amigos que se encuentran empleados se tornan en una fuente de información o recomendación: así ocurrió con el 45,9 \% de los egresados, quienes accedieron a puestos de trabajo a partir de las relaciones que cultivaron durante el estudio de la carrera. De igual forma, expresaron servir 

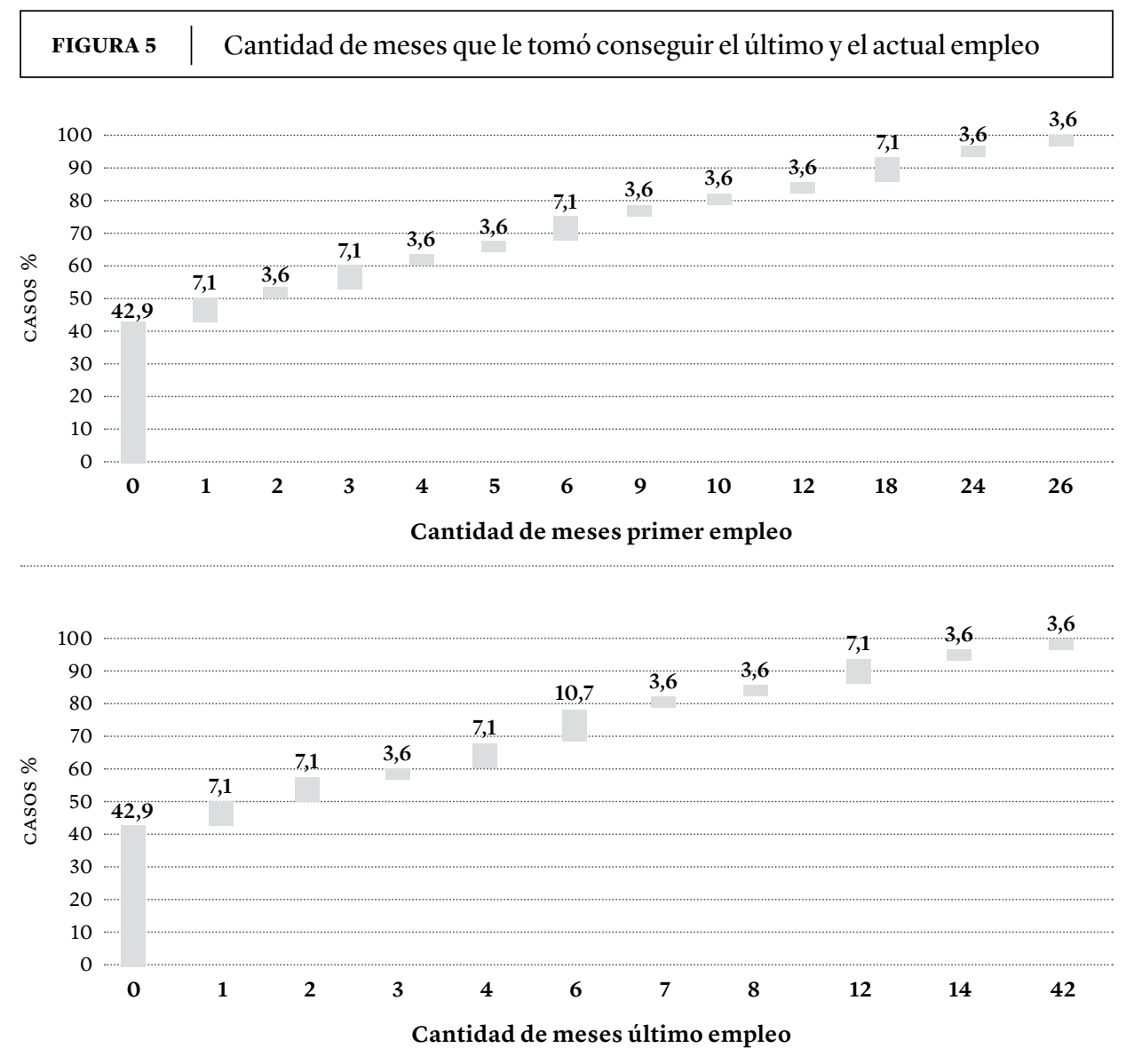

Fuente: elaboración propia.

como canal para otros de sus colegas, a quienes les remitían información de interés relacionada con empleos puesto que se encontraban ya ocupados, o porque la labor no correspondía al perfil de trabajo buscado.

Solo el 8,3\% de la población indicó que accedió a empleos por medio de un familiar que conocía personas con influencia para facilitar la inserción en puestos de trabajo profesionales; en tales casos, las relaciones constituyeron el factor definitivo para superar dificultades de acceso al empleo tales como la falta de experiencia, lo cual evidencia que el título profesional no es recurso suficiente para lograr un ejercicio efectivo de la profesión en los casos estudiados.

Es importante anotar que los empleos obtenidos por $54,5 \%$ de los encuestados les han permitido poner en práctica todos o casi todos los conocimientos adquiridos en el 
pregrado; y al 27,3\%, cerca de la mitad. Las ocupaciones y actividades se encuentran relacionadas, principalmente, con procesos comunitarios y ejercicio de la docencia, labores que, según ellos, les han permitido tener una nueva comprensión del mundo y nuevos entornos de aprendizaje en el campo de la profesión sociológica. Todo ello refuerza en unos casos y resignifica en otros el conocimiento recibido en la universidad. En otras palabras, los egresados han logrado emplearse en cargos en los que el perfil profesional ha resultado idóneo frente a las necesidades del mercado laboral.

Respecto a las mayores dificultades que han vivido este grupo de profesionales de la Sociología en el ejercicio de su carrera, un aspecto significativo tiene que ver con la existencia de campos de acción con límites cada vez más difusos entre las diferentes disciplinas de las ciencias sociales: ámbitos como la intervención o acción social comunitaria dejan de ser exclusivos de un área en particular y causan una disputa con egresados de Trabajo Social, Antropología o Psicología, entre otras carreras, fenómeno registrado en otras investigaciones (Castillo-Riquelme; Rodríguez-Garcés, 2018). Frente a las dificultades causadas por no tener un campo de acción exclusivo, los egresados manifestaron que compartir de forma interdisciplinaria les ha posibilitado un proceso más integral, de un constante aprendizaje en el ejercicio profesional.

A lo anterior se suman otras situaciones, tales como la poca estabilidad laboral a causa de las características de los contratos: $36,5 \%$ de los graduados laboran bajo contratación a término fijo inferior a un año; y el $25 \%$, en la modalidad de prestación de servicios. Si bien estos contratos traen los beneficios de tener estabilidad laboral por el tiempo acordado y contar con las prestaciones establecidas por la ley-solo en el primer caso-, a la vez que con ventajas relacionadas con la autonomía de tiempo en el desarrollo de sus funciones y una libertad que permite acceder a otros espacios laborales -en el segundo caso-, permanecen en incertidumbre laboral al no contar con un puesto a término indefinido.

No obstante estas dificultades, los egresados manifiestan haber logrado una mejora considerable en sus ingresos económicos, al tener en cuenta que antes del acceso a la universidad estos estaban sustentados por sus núcleos familiares donde los ingresos del grupo oscilaban entre 1 y 3 salarios mínimos legales vigentes (SMLV), y que para el titulado evidenciaron una variación significativa dado que, en la misma escala de medición, los recursos devengados por la mayor parte de ellos corresponden a un monto que oscila entre 3 y $5 \mathrm{SMLV}$ (figura 6). A este respecto es importante señalar que los ingresos de las familias se disponían para los gastos de todos los integrantes del núcleo familiar; mientras que en el caso de los egresados, la destinación de sus recursos se da en mayor proporción para sus gastos personales, lo cual constituye, según las narraciones de sus experiencias, una mejora significativa 
en sus condiciones materiales individuales y en sus hogares. Al respecto, 64,2 \% manifestó apoyar de forma permanente o en muchas ocasiones a su grupo familiar de origen, mientras que $21,4 \%$ lo ha hecho con mediana frecuencia.

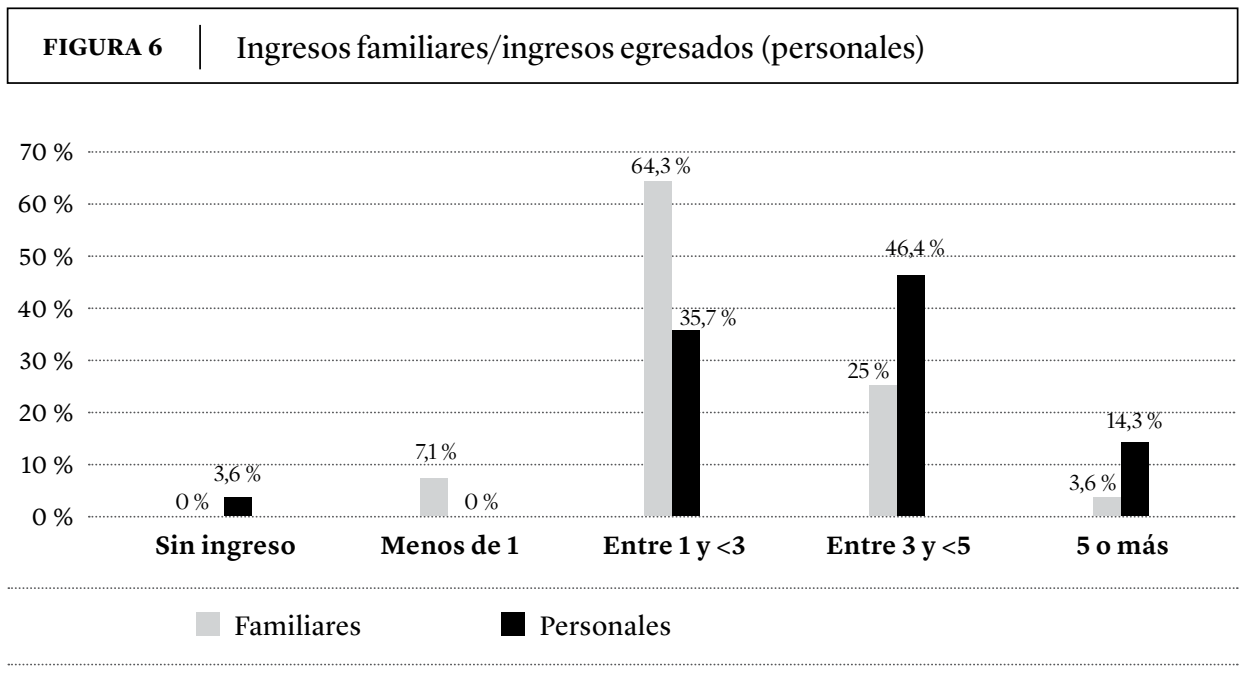

Fuente: elaboración propia.

La mejora a nivel individual también se ve reflejada en las prácticas de consumo de los egresados: 39,3\% manifestó haber adquirido una vivienda, e igual porcentaje compró un vehículo. Además, se encontró un incremento en actividades de ocio como viajes (locales e internacionales) y visitas a restaurantes y cines frente a las realizadas antes de profesionalizarse (figura 7); ello cual evidencia cambios en el estilo de vida, conducentes a mejorar la posición social (Bonnewitz, 2006). Estas modificaciones, según los egresados, se deben al proceso formativo, que propició una transformación en su percepción sobre las cosas, haciéndolos personas más conscientes acerca del uso del tiempo libre como un aspecto ligado al desarrollo personal y social, el cual representa un progreso material y de las condiciones de vida no materiales.

De lo anterior se desprende que la formación superior brindó a los egresados la oportunidad de adquirir nuevos recursos de conocimiento y relaciones al materializar las motivaciones en logros de diferente tipo. Por un lado, les permitió modificar las condiciones de su origen social; y por otro, les otorgó el poder de influir en diferentes miembros del grupo familiar - cercano y extenso- y en algunos círculos de amigos en lo que respecta a las decisiones que toman, los contenidos de las relaciones en diferentes ámbitos e, incluso, la orientación de las acciones desarrolladas, aspec- 


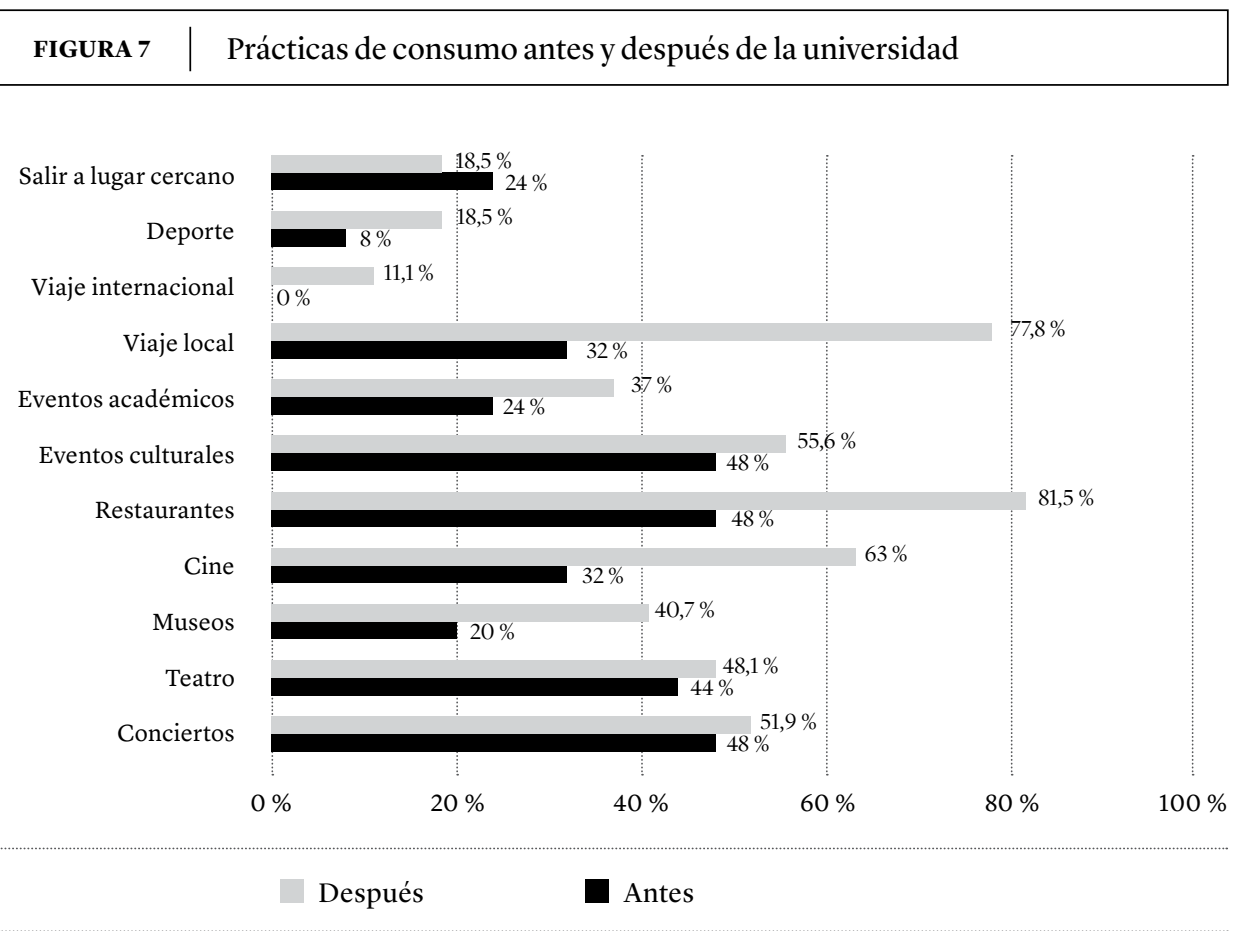

Fuente: elaboración propia.

tos en los cuales no tienen injerencia significativa antes de tener un recorrido por lo universitario. En otras palabras, han logrado reconocimiento profesional en sus grupos de pertenencia cercanos.

\section{Logro educativo como transformador de los vínculos familiares y personales}

Un aspecto relevante en la narración de la experiencia de los egresados, derivado de su formación profesional, se relaciona con la ubicación como referentes para sus familias en diferentes dimensiones. Recopilan y entretejen elementos de su experiencia personal para dirigir y motivar los procesos educativos de otros integrantes de su núcleo de crianza; por ejemplo, narraron que primos o hermanos se han visto alentados a emprender y terminar la formación superior universitaria. Además, debido a los conocimientos que adquirieron en Sociología, quedaron acreditados para dar opiniones y orientar decisiones de todo tipo dentro de este mismo ámbito (figura 8). Esto se traduce en el fortalecimiento de vínculos para el 32,1 \% de los 
casos, aunque durante la carrera haya significado choques y rompimientos debido a las posiciones encontradas frente a creencias religiosas, políticas y hábitos de vida representados en los cambios de percepciones e intereses, lo cual fue reconocido por el $46,4 \%$ de la población abordada.

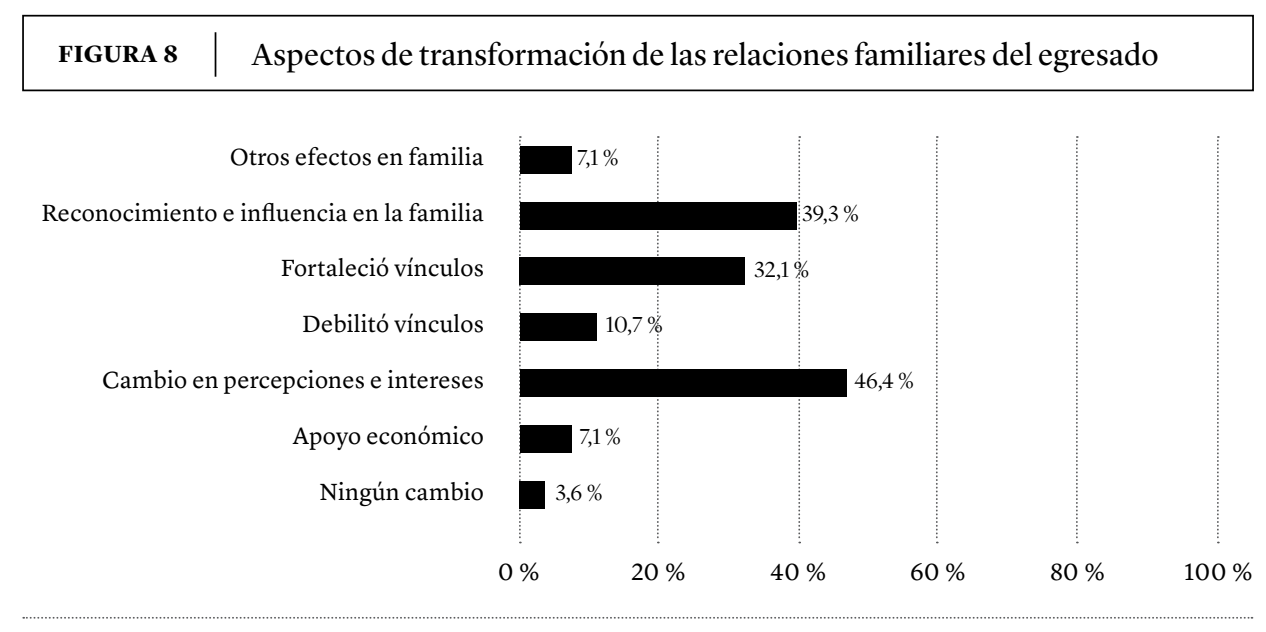

Fuente: elaboración propia.

Este escenario de transformación, que contiene admiración y reconocimiento debido al logro de nuevos aprendizajes, los cuales eran ajenos y hasta imposible de alcanzar para los grupos familiares, fortalecen, comparten y entretejen relaciones, estilos de vida o gustos, creencias y prácticas, opiniones políticas, convicciones y preferencias, así como hábitos de todo tipo -alimenticios y culturales, entre otros-. Es decir, se trata de un modo de vida "(...) que corresponde a la cantidad de bienes y servicios de los cuales puede disponer un individuo o un grupo" (Bonnewitz, 2006: 69): para el caso, el egresado y su familia.

Como lo señalan algunos de los encuestados, se han constituido contextos que han llevado a la modificación de perspectivas frente a visiones e ideas que se tenían instauradas, y fueron recurrentes durante toda la trayectoria educativa. La educación superior no solo era vista como algo inalcanzable y no valorado por parte de los hogares en los que no existía educación universitaria; además, para el caso específico del pregrado en Sociología, se veía como una carrera subvalorada en cuanto a su funcionalidad en la sociedad y a asuntos políticos-situación presentada en la mayoría de los titulados-, y como una profesión ligada a una carga ideológica que la denotaba conforme a pensamientos de izquierda, aspectos mal vistos en sus 
ambientes más cercanos y anclados a visiones compartidas en el mundo laboral, y en general, de la sociedad.

Desde la experiencia narrada, el proceso educativo estuvo determinado por nuevos relacionamientos que permitieron construir redes estables, las cuales trascendieron en vínculos de amistad conservados con compañeros de la universidad -del mismo y de otros pregrados-y que representan estructuras de apoyo mutuo en situaciones económicas difíciles, en la realización de trabajos dentro y fuera del espacio universitario, y en sí, en apoyos personales para la vida diaria de los egresados.

En consonancia con lo anterior, desde los discursos se reconocen algunos casos en los cuales los egresados encontraron limitaciones en sus relacionamientos o en la ampliación de redes, debido a que en muchas de las experiencias, y por ser estudiantes trabajadores, no contaban con tiempo para interactuar o participar en espacios de socialización con otras personas pertenecientes a la institución, es decir, con los mismos compañeros, funcionarios administrativos y profesores. Algunos indicaron ser solitarios y ensimismados, situaciones que causaban dos situaciones: de un lado, que no se generara una armonización en el actuar con otros; y de otro, que al egresar del campo educativo encontraran dificultades con ciertos elementos o carecieran de ellos al emprender sus roles como profesionales, esto es, frente a los vínculos para el trabajo y la cercanía con otras personas para lograr acompañamiento.

\section{Conclusiones}

Tras el logro educativo y lo que acarrea -la entrada a la universidad, el transcurso de la vida educativa y, luego, el ejercicio profesional-, los egresados se vieron sujetos a transformaciones significativas en el conjunto de relaciones sociales que han mantenido y construido en el curso de sus vidas (Bonnewitz, 2006), esto es, en posiciones y vínculos familiares que, desde su llegada a la institución de educación superior, se vieron dirigidos no solo hacia el cambio del nivel educativo por parte del egresado, sino a un cúmulo de situaciones, de encuentros y desencuentros, de influjos y de apoyos que, al interior del espacio familiar, fundaron una nueva experiencia. Esta se caracteriza por situaciones emergentes, tales como la motivación por estudiar que se instala en las generaciones contemporáneas y posteriores a los egresados, así como por la modificación de las visiones y perspectivas que se tenían en los núcleos familiares sobre el papel del profesional de la Sociología en la sociedad.

Sumado a lo anterior, la experiencia educativa aportó nuevos relacionamientos más allá de los hogares, que se traducen en la consecución de nuevos amigos, parejas y compañeros interdisciplinares con los cuales los egresados lograron ampliar 
$\mathrm{y}$ fortalecer redes de acompañamiento y laborales. Ello constituye un espectro de socialización que los ha llevado a tener lazos duraderos, a la vez que conexiones útiles (Bourdieu, 2011) para el ejercicio profesional, así como para sentirse satisfechos en diferentes aspectos en el desarrollo de sus proyectos de vida y en la configuración de roles sociales. Haber atravesado un proceso de aprendizaje en un programa específico -Sociología-les brindó una formación teórica y metodológica para afrontar y aportar a un sinnúmero de problemáticas y transformaciones, tales como la violencia, el desplazamiento y la creciente industrialización y urbanización, entre muchos otros.

Las motivaciones en los egresados estuvieron determinadas no solo por el accionar individual (orientado por la búsqueda de cambiar situaciones personales y condiciones de vida de origen o por el deseo de potencializar su sentido con lo social), sino también por las oportunidades de acceso ofrecidas por la UdeA. Estos elementos aportaron a la transformación de sus relaciones, roles, proyectos de vida y visiones de futuro (Avendaño-Castro; Paz-Montes; Rueda-Vera, 2017), las cuales estaban mediadas por condiciones contextuales (Guzmán-Bracho, 2019). Los egresados vieron en la educación universitaria una oportunidad de alcanzar la realización personal (Guzmán-Gómez, 2017), así como de fortalecer y ampliar sus saberes, dado que en su mayoría provenían de familias con poco capital cultural acumulado, debido a dos factores discutidos en secciones previas: el ejercicio de oficios no cualificados por parte de las personas encargadas de la crianza; y el desarrollo de pocas actividades intelectuales. Sobre esto, Bourdieu y Passeron (1996) argumentan que las clases populares han tenido más posibilidades de eliminarse en la enseñanza secundaria puesto que existe un componente-el examen-que les representa un posible fracaso frente a la realización de estudios superiores, toda vez que no logran ingresar con facilidad a los centros académicos.

Pese a lo anterior, el acceso se posibilitó gracias a la política de descentralización educativa planteada por la institución universitaria estudiada en cumplimiento de la política pública de educación superior, orientada a una educación que brinde de elementos como la generación y la crítica de ideas. Estos se hicieron evidentes al establecer relacionamientos y ejercer la profesión, y constituyen recursos que no dependen de forma directa de la posesión de un capital cultural heredado o de la carga del origen social ${ }^{1}$, la cual ha disminuido con la expansión educativa.

Conforme avanzaron en su plan de estudios, los que después serían egresados acumularon nuevos conocimientos, amigos, experiencias y prácticas, y una titulación que les generó satisfacción en lo personal, económico, profesional y laboral, por disponer de un diploma que "es elemental para asegurar una ventaja decisiva en 
la competencia profesional" (Bourdieu; Passeron, 1996: 233). Tales componentes, interiorizados en el marco del proceso de la enseñanza y el aprendizaje (Bonnewitz, 2006), al obtener el título y emprender el camino laboral también disminuyeron la preponderancia del origen (Marqués-Perales; Gil-Hernández, 2015), al convertir el grado en un requerimiento para acceder al posicionamiento ocupacional relacionado con su formación académica, en el que ponen en práctica fundamentos teóricos $\mathrm{e}$ históricos, de trabajos con comunidades, de análisis y críticas, y de buscar y adquirir contactos. Es decir, una capacidad mediante la acción que ellos -los egresadosemprendieron, que los llevó a tener cambios y posicionarse como referentes de sus grupos cercanos debido a las condiciones que generan un mayor nivel de escolaridad intergeneracional (Rodríguez, 2016), así como a los diferentes relacionamientos que pudieron establecer en razón de sus conocimientos, de la transformación de sus condiciones de vida y de las actividades emprendidas para efectuar dichos cambios.

Las acciones y logros de estos egresados también tuvieron efectos importantes en sus núcleos cercanos. Por ejemplo, en muchos casos lograron la transición de ideas que su núcleo familiar tenía sobre la formación en Sociología: predominó una estigmatización durante el transcurso de todo su proceso educativo por creer que este programa no tenía el mismo valor que carreras de otras facultades o con otros contenidos (Baltar; Siqueira-Baltar, 2017), o que estaba ligada a preceptos ideológicos. Esta concepción cambia cuando el egresado logra estabilidad en lo personal y lo profesional, y establece nuevas redes con amigos y colegas que se encuentran en su mismo nivel educativo.

Estas redes se consolidan como vínculos útiles para el conocimiento y reconocimiento mutuos (Bourdieu, 2011), y se comportan como eslabones fundamentales para la consecución de nuevos empleos. Aportan así a las transformaciones frente al entorno familiar de los egresados y su crecimiento en términos intelectuales, profesionales y económicos; además, brindan la posibilidad de pertenecer a otros grupos exclusivos de órdenes académico y cultural, entre otros.

Los resultados presentados permiten comprender que la evaluación de la política pública educativa, al hacer énfasis en la de educación superior, debe ir más allá de la verificación de su cumplimiento en términos de acceso, competencias y vínculos con el mercado laboral-aunque sin excluirlos-. Esto por cuanto su impacto se evidencia en la transformación de la experiencia de los egresados y grupos más cercanos, situación que configura y da un giro al fundamento de sus proyectos de vida a largo plazo -es decir, el cumplimiento de metas y el accionar de cada uno-, con lo cual se logra impactar y transformar varias generaciones y círculos cercanos a ellos. 


\section{Referencias}

Aguilar-Gaviria, Sonia; Barroso-Osuna, Julio (2015). La triangulación de los datos como estrategia en investigación educativa. Pixel-Bit. Revista de Medios y Educación, 47, 73-88.

Alzás-García, Teresa; Casa-García, Luis Manuel (2017). La evolución del concepto de triangulación en la investigación social. Revista Pesquisa Qualitativa, 5(8), 395-418. Recuperado de https://www.researchgate.net/publication/320700886_La_evolucion_del_ concepto_de_triangulacion_en_la_investigacion_social

Avendaño-Castro, William; Paz-Montes, Luisa; Rueda-Vera, Gerson (2017). Políticas públicas y educación superior: análisis conceptual del contexto colombiano. Revista Venezolana de Gerencia, 22(79), 1-20. Recuperado de https://www.redalyc.org/ jatsRepo/290/29055964008/html/index.html

Baltar, Ronaldo; Siqueira-Baltar, Claudia (2017). A Sociologia como profissão. Revista Brasileira de Sociología, 5(10), 259-289. http://dx.doi.org/10.20336/rbs.213

Beltrán, William Mauricio; Berrío-Gil, Darío (2016). Viviendo de la Sociología: egresados(as) de la Universidad Nacional de Colombia y mercados parciales de trabajo. Sociedady Economía, (30), 121-146. Recuperado de http://www.scielo.org.co/pdf/soec/n3o/n3oao6.pdf

Beltrán, William Mauricio; Torres-Ortiz, María Raquel (2016). Calidad y pertinencia del Programa Curricular de Sociología de la Universidad Nacional de Colombia desde la perspectiva de sus egresados. Revista Colombiana de Sociología, 38 (2), 139-165. Recuperado de https://revistas.unal.edu.co/index.php/recs/article/view/55551/54837

Blanco, Neligia; Pirela, Johann (2016). La complementariedad metodológica: estrategia de integración de enfoques en la investigación social. Espacios Públicos, 19(45), 97-111. Recuperado de https://www.redalyc.org/jatsRepo/676/67646966005/html/index.html

Bonnewitz, Patrice (2006). La Sociología de Pierre Bourdieu. Buenos Aires: Nueva Visión.

Bourdieu, Pierre; Passeron, Jean-Claude (1996). La reproducción. Elementos para una teoría del sistema de enseñanza. México: Editorial Laia. Recuperado de https://socioeducacion.files. wordpress.com/2011/o5/bourdieu-pierre-la-reproduccion1.pdf

Bourdieu, Pierre (2001). Poder, derechoy clases sociales. Bilbao: Desclée de Brower. Recuperado dehttps://erikafontanez.files.wordpress.com/2015/o8/pierre-bourdieu-poder-derecho-yclases-sociales.pdf

Bourdieu, Pierre (2011). Las estrategias de la reproducción social. Buenos Aires: Siglo Veintiuno Editores. 
Castillo-Riquelme, Víctor; Rodríguez-Garcés, Carlos (2018). Los problemas del stock en campos profesionales difusos: Oferta educativa en Trabajo Social. Estudios Pedagógicos, 42(1), 37-52. https://www.doi.org/10.4067/So718-07052016000100003

Departamento Nacional de Planeación - DNP (s. f.). Bases del Plan Nacional de Desarrollo 2014-2018. Recuperado de https://colaboracion.dnp.gov.co/CDT/Prensa/PND\%2O2O142018\%20Bases\%2oFinal.pdf

Departamento Nacional de Planeación - DNP (2018). Plan Nacional de Desarrollo 2018-2022: pacto por Colombia, pacto por la equidad. Recuperado de https://colaboracion.dnp.gov.co/ CDT/Prensa/Resumen-PND2018-2022-final.pdf

Departamento Nacional de Planeación - DNP (2019). Bases del Plan Nacional de Desarrollo 2018-2022. Recuperado de https://colaboracion.dnp.gov.co/CDT/Prensa/BasesPND2O182022n.pdf

Diez, María Agustina (2017). La Sociología como profesión: desencuentros entre la formación académica y la inserción laboral. Cuadernos de pesquisa, 47(165), 912-937. https://doi. org/10.1590/198053144355

Díaz, Claudio; Galán, Gabriela (2015). Caracterización de estudiantes de nutrición de la Universidad del Mar de Talca según composición de capital cultural y social. Revista chilena de nutrición, 42(1), 53-59. http://dx.doi.org/10.4067/So717-75182015000100007

Facchini, Carla (2019). Employment and Education of Sociologists. Opportunities and Critical Factors of a Multifaceted Profession International. Review of Sociology, 29(3), 327-335. https:// doi.org/10.1080/03906701.2019.1672348

Fernández-Esquinas, Manuel (2019). Dealing with the Profession of Sociology: Opportunities and Problems at the Interface Between Training and Professional Development. International Review of Sociology, 29(3), 336-353. https://doi.org/10.1080/03906701.201 9.1672349

García-Yepes, Karen (2017). Construcción de proyectos de vida alternativos (PVA) en Urabá, Colombia: papel del sistema educativo en contextos vulnerables. Estudios pedagógicos (Valdivia), 43(3), 153-173. https://dx.doi.org/10.4067/So718-07052017000300oo9

Giddens, Anthony (2006). La constitución de la sociedad: bases para la teoría de la estructuración $3 .{ }^{a}$ ed. Buenos Aires: Amorrortu.

Gómez-Urrutia, Verónica; Royo-Urrizola, Paulina (2015). Nuevas subjetividades y proyectos de vida: jóvenes universitarios de la VII región de Maule, Chile. Revista de Estudios Sociales, 53, 90-101. http://dx.doi.org/10.7440/res53.2015.07

González, Gabriel; Díez-Echavarría, Luisa; Zapa, Elkin; Eusse, Danilo (2019). Evaluación del impacto académico y social en egresados universitarios. Revista Complutense de Educación, 30(3), 695-712. https://doi.org/10.5209/rced.57774 
Granovetter, Mark (1973). La fuerza de los vínculos débiles. American Journal of Sociology, 78 , 1360-1380.

Granovetter, Mark (1995). Getting a Job. A Study of Contacts and Careers. Chicago and London: University of Chicago Press.

Gutiérrez, Jahir; Mondragón, Viviana; Santacruz, Laura (2019). Expectativas, necesidades y tendencias de la formación en educación superior en Colombia en pregrado y posgrado: entre la deserción-perfil y vocación profesional. Universidad y Empresa, 21(37), 313-345. http://dx.doi.org/10.12804/revistas.urosario.edu.co/empresa/a.6619

Guzmán-Bracho, Mauricio (2019). Agencia Constructiva: acción social para el bienestar colectivo. Iberóforum. Revista de Ciencias Sociales de la Universidad Iberoamericana, XIII(26), 1-27. Recuperado de https://www.redalyc.org/journal/2110/211059782001/html/

Guzmán-Gómez, Carlota (2017). Las nuevas figuras estudiantiles y los múltiples sentidos de los estudios universitarios. Revista de la Educación Superior, 46(182), 71-87. https://doi. org/10.1016/j.resu.2017.03.002

Lomelí-Parga, Andrea Magdalena; López-Padilla, María Guadalupe; Valenzuela-González, Jaime Ricardo (2016). Autoestima, motivación e inteligencia emocional: Tres factores influyentes en el diseño exitoso de un proyecto de vida de jóvenes estudiantes de educación media. Revista Electrónica Educare, 2O(2), 1-22. http://dx.doi.org/10.15359/ree.2O-2.4

López-Cárdenas, Irina; Mella-Luna, Julio; Cáceres-Valenzuela, Gabriela (2018). La universidad como ruptura en la trayectoria educativa: experiencias de transición de estudiantes egresados de Enseñanza Media Técnico Profesional que ingresan al Programa Académico de Bachillerato de la Universidad de Chile. Estudios pedagógicos, 44(3), 271-288. http:// dx.doi.org/10.4067/So718-07052018000300271

Malagón-Plata, Luis Alberto; Rodríguez-Rodríguez, Luz Helena; Machado-Vega, Diego Fernando (2019). Políticas públicas educativas y aseguramiento de la calidad en la educación superior. Revista Historia de la educación Latinoamericana, 21(32), 273-290. https://doi.org/10.19053/01227238.4999

Marqués-Perales, Ildefonso; Gil-Hernández, Carlos (2015). Origen social y sobreeducación en los universitarios españoles: ¿es meritocrático el acceso a la clase de servicio? Revista Española de Investigaciones Sociológicas, 150, 89-112. http://dx.doi.org/10.5477/cis/reis.150.89

Ministerio de Educación Nacional - MEN (s. f.). Lineamientos Política de Educación Superior Inclusiva e intercultural. Recuperado de https://www.mineducacion.gov.co/1759/w3article-357277.html?_noredirect=1

Ministerio de Educación Nacional - MEN (s. f.). Sistema educativo colombiano. Recuperado de https://www.mineducacion.gov.co/portal/Educacion-superior/Sistema-de-EducacionSuperior/231235:Sistema-Educativo-Colombiano\%C3\%A7 
Navarro-Leal, Esteban; Sembler-Reyes, Matías (2018). "No nos sobra, pero por eso trabajamos": movilidad social intergeneracional e inconsistencia posicional en asistentes de aula y asistentes de párvulo. Revista Némesis, 14, 13-40. Recuperado de https://dialnet.unirioja. es/servlet/articulo?codigo $=7084615$

Oriente iniciará en 2004 con sede propia (2003). Alma Mater, 29.

Puentes-Borges, Abundio-Eduardo; Puentes-Bencomo, Digna Beatriz; Puentes-Bencomo, Eduardo Rangel (2017). Por mayor objetividad en la triangulación de los instrumentos de diagnóstico. Didácticay Educación, 8(4), 77-84. Recuperado de https://dialnet.unirioja.es/ servlet/articulo? codigo $=6681318$

Rodríguez, Santiago Andrés (2016). Logros educativos en el nivel de instrucción superior y movilidad educacional intergeneracional en Argentina. Sociológica, 31(88), 167-200. Recuperado de http://www.scielo.org.mx/pdf/soc/v31n88/o187-0173-soc-31-88-o0167.pdf

Sánchez, María Cruz (2015). La dicotomía cualitativo-cuantitativo: posibilidades de integración y diseños mixtos. Campo Abierto. Revista de Educación, 1, 11-30. Recuperado de https:// libros.unlp.edu.ar/index.php/unlp/catalog/view/582/546/1968-1

Trindade, Victoria Andrea (2016). Entrevistando en investigación y los imprevistos en el trabajo de campo: de la entrevista semiestructurada a la entrevista no estructurada. En Técnicas y estrategias en la investigación cualitativa (pp. 18-34), coordinado por Patricia Schettini e Inés Cortazzo. La Plata: Universidad de la Plata.

Universidad de Antioquia - UdeA (2017). Plan de desarrollo 2017-2027, una universidad innovadora para la transformación de los territorios. Recuperado de http://www2.udea.edu. co/webmaster/multimedia/plan-desarrollo-udea/plan-desarrollo-udea.pdf

Universidad de Antioquia - UdeA (2020). UdeA en las regiones. Recuperado de http://www.udea. edu.co/wps/portal/udea/web/inicio/udea-regiones/oriente/contenido/asmenulateral/ egresados/!ut/p/z1/vVVNc5swFPwrzoEjlviG3ih23TpgwDENcOnIIGM6IBEh25n-

Vázquez-González, Natalia Ix-chel; Díaz-Pérez, Guillermina; Pérez-Damian, Araceli (2015). La sombra de la violencia estructural en los jóvenes universitarios. Recerca, 16, 59-86. https:// doi.org/10.6035/Recerca.2015.16.4

Veliz-Rojas, Lizet; Bianchetti-Saavedra, Andrés (2016). Integración metodológica en la investigación de fenómenos complejos en enfermería. Index de Enfermería, 25(1-2), 47-50. Recuperado de http://scielo.isciii.es/scielo.php?script=sci_ arttext\&pid=S1132-12962016000100011

Vieytes, Rut (2004). Metodología de la investigación en organizaciones, mercado y sociedad: epistemología y técnicas. Buenos Aires: Editorial de las Ciencias.

Yaschine, Iliana (2015). ¿Alcanza la educación para salir de la pobreza? Análisis del proceso de estratificación ocupacional de jóvenes rurales en México. Revista Mexicana de Ciencias Politicas y Sociales, LX(223), 377-406. https://doi.org/10.1016/So185-1918(15)72142-2 


\section{Anexos}

ANEXO 1 $\mid \begin{aligned} & \text { Red semántica del tema Logro personal como fundamento } \\ & \text { dela motivación para profesionalizarse }\end{aligned}$

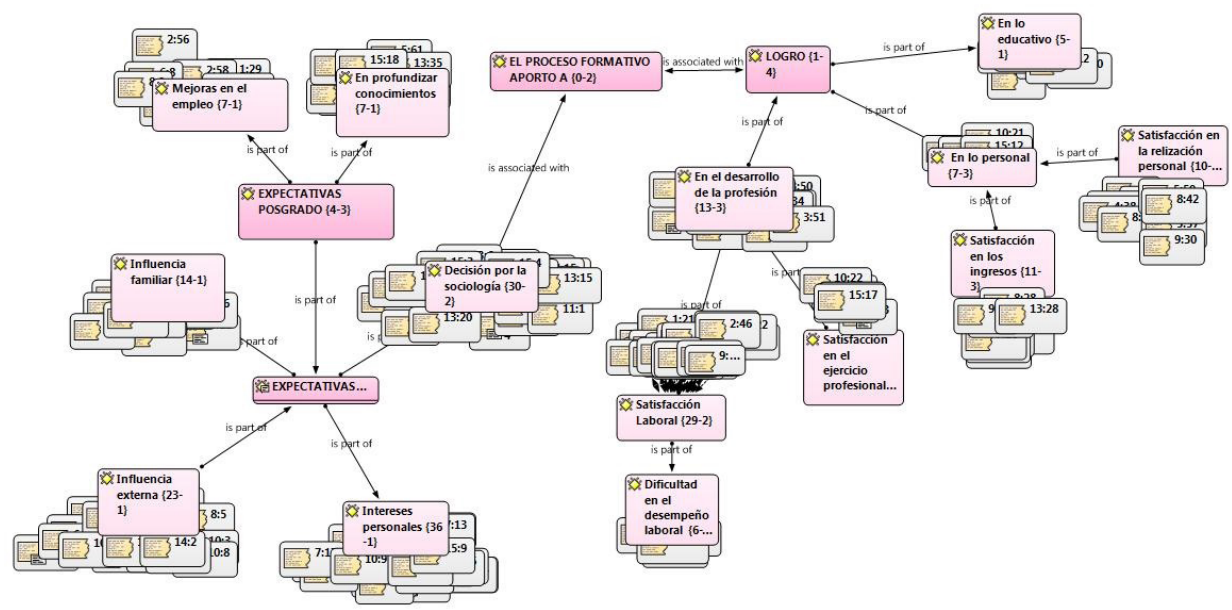




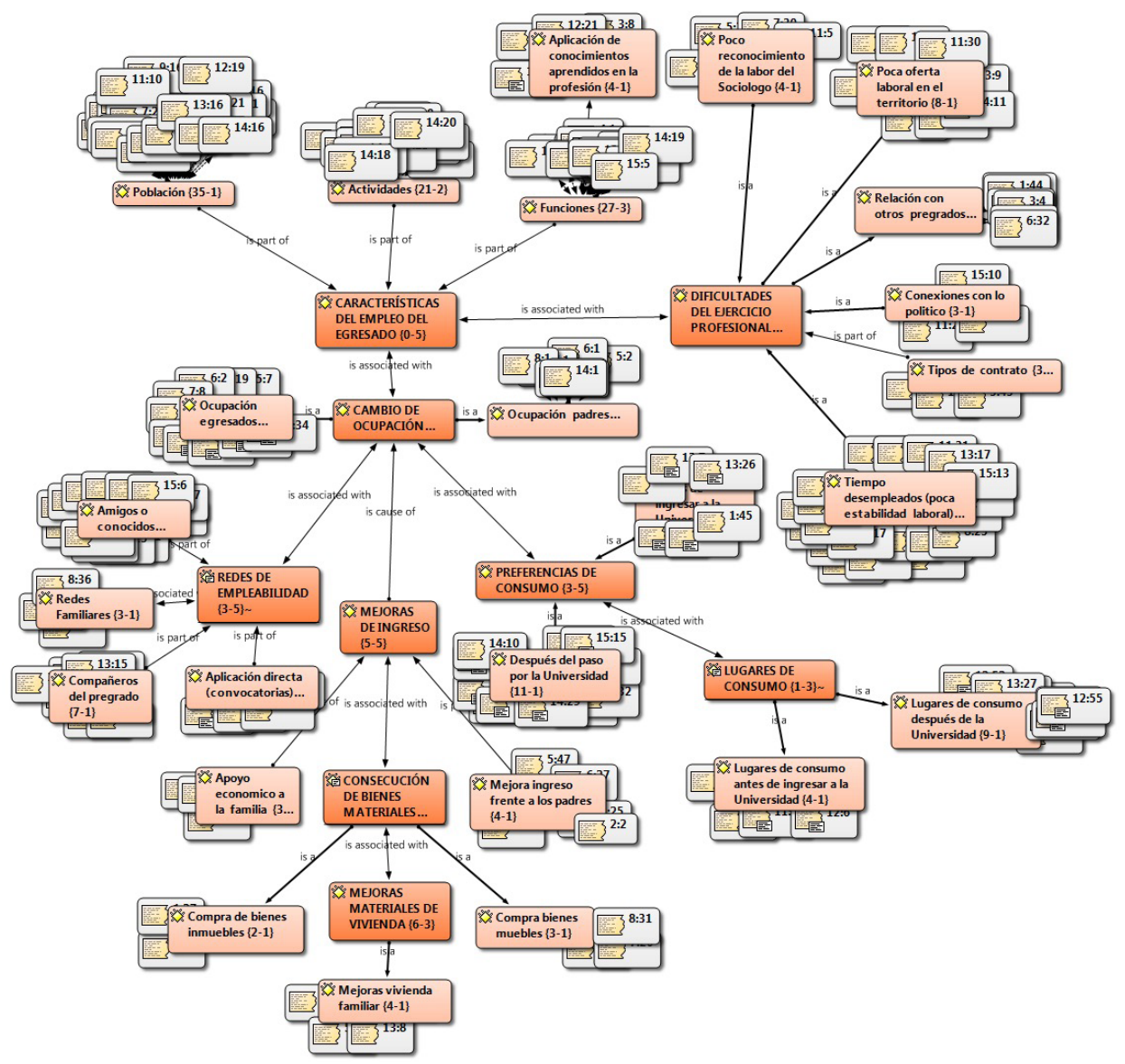




\section{ANEXO 3 Red semántica del tema Logro educativo como transformador de los vínculos familiaresy personales}

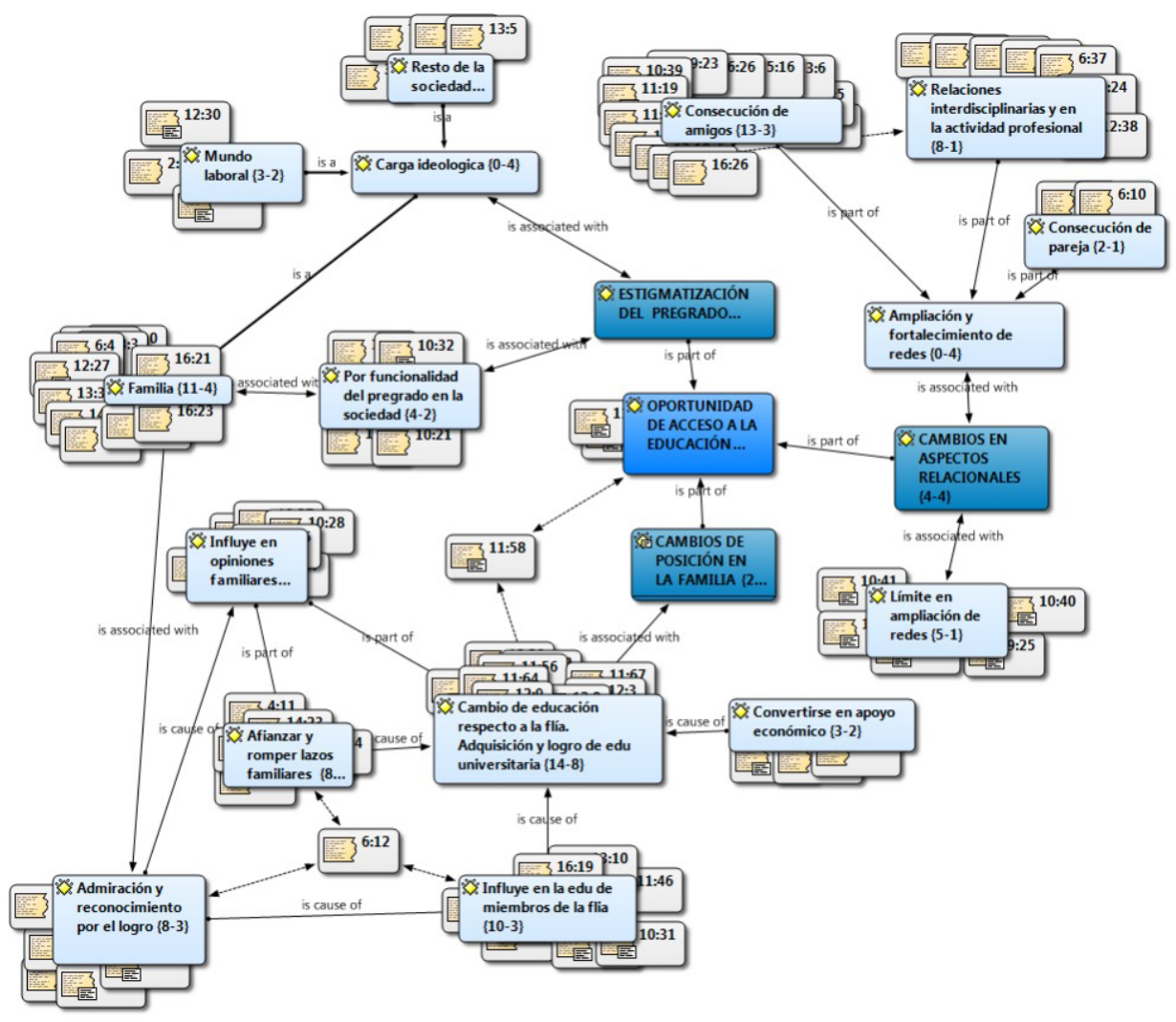

